\title{
Übersterblichkeit heute kein generelles Phänomen mehr
}

Fragestellung: Gilt das Paradigma der Übersterblichkeit von Typ2-Diabetikern im Vergleich zur Allgemeinbevölkerung grundsätzlich und für alle Konstellationen oder gibt es Ausnahmen?

Hintergrund: Nach der Lehrmeinung ist bei allen Menschen mit Diabetes die Gesamtmortalität und die Sterblichkeit infolge kardiovaskulärer Ursachen erhöht. Kürzlich wurde gezeigt, dass diese bei Typ-1-Diabetikern mit guter Stoffwechseleinstellung $\left(\mathrm{HbA}_{1 \mathrm{c}}\right.$ unter 7,0\%) auch heute noch um das Zweifache erhöht sind. Für Typ-2-Diabetiker, bei denen außer der Stoffwechselkontrolle zusätzlich behandelbare Risikofaktoren wie Übergewicht, Hypertonie und Hyperlipidämie hohe prognostische Bedeutung haben, fehlen solche Untersuchungen bislang.

\section{Originalie}

Tancredi M, Rosengren A, Svensson A-M et al. Excess Mortality among Persons with Type 2 Diabetes. New Engl J Med. 2015;373:1720-32.
Patienten u. Methoden: Von 1998 -2011 wurden in Schweden 435.369 Typ-2-Diabetiker registriert. Sie wurden 1:5 mit 2.117.483 alters-, geschlechtsu. herkunftsgleichen Personen aus der Allgemeinbevölkerung gegenübergestellt.
Ergebnisse:

1. Innerhalb von 4,7 Jahren starben 77.117 (17,7\%) Diabetiker und 306.097 (14,5\%) Kontrollpersonen. Die entsprechenden Raten für die kardiovaskuläre Mortalität betrugen 7,9 bzw. 6,1\% (absolute Differenz 18 bzw. 23\%).

2. Demgegenüber liegt die Übersterblichkeit bei Diabetikern unter 55 Jahren mit schlechter Stoffwechselkontrolle und mit Nephropathie 200 bis $300 \%$ höher.

3. Im Umkehrschluss sind Gesamt- und kardiovaskuläre Sterblichkeit bei alten Diabetikern ( $\geq 75$ Jahre) mit guter Stoffwechselkontrolle $(0,95)$ und bei solchen $\geq 65$ Jahre ohne Nephropathie $(0,92)$ nicht nur niedriger als bei Patienten unter 55 Jahren (1,92 bzw. 2,18), sondern auch im Vergleich zu Kontrollpersonen (Referenzwert 1,0).

Schlussfolgerung: Die Gesamtsterblichkeit und die kardiovaskuläre Mortalität von Patienten mit Typ-2-Diabetes im Vergleich zur Allgemeinbevölkerung schwanken in Abhängigkeit von Alter, von Stoffwechselkontrolle und Nephropathie ganz erheblich zwischen Übersterblichkeit und sogar niedrigerem Sterberisiko.

\section{- Kommentar von Prof. Dr. med. Heinrich Holzgreve}

\section{Lehrmeinung modifiziert - Unterschiede beträchtlich}

Im Gesamtkollektiv erreicht die Übersterblichkeit von Diabetikern im Vergleich zur Allgemeinbevölkerung ein historisches Tief von ca. 20\%. Die Autoren führen diese Entwicklung auf die Therapie mit Antidiabetika, Lipidsenkern und Antihypertensiva zurück, die bei Diabetikern häufig aggressiver erfolgt als bei Nichtdiabetikern. Allerdings sind die Unterschiede in diversen Untergruppen beträchtlich. So haben jüngere Patienten mit schlechter Stoffwechselkontrolle und Nephropathie weiterhin eine 2- bis 3-fach höhere Sterblichkeit, während bei älteren Pa- tienten mit guten $\mathrm{HbA}_{1 c}$-Werten und Kontrolle der Risikofaktoren keine höhere Mortalität im Vergleich zur Allgemeinbevölkerung mehr nachweisbar ist. Diese Ergebnisse rütteln an der verbreiteten Ansicht, die allen Diabetikern pauschal eine Übersterblichkeit zuschreibt.

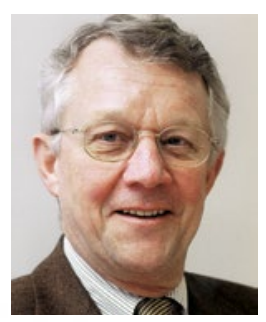

Prof. Dr. med. Heinrich Holzgreve

Internist, Kardiologische Praxis

Burgstr. 7, 80331 München

h.holzgreve@t-online.de

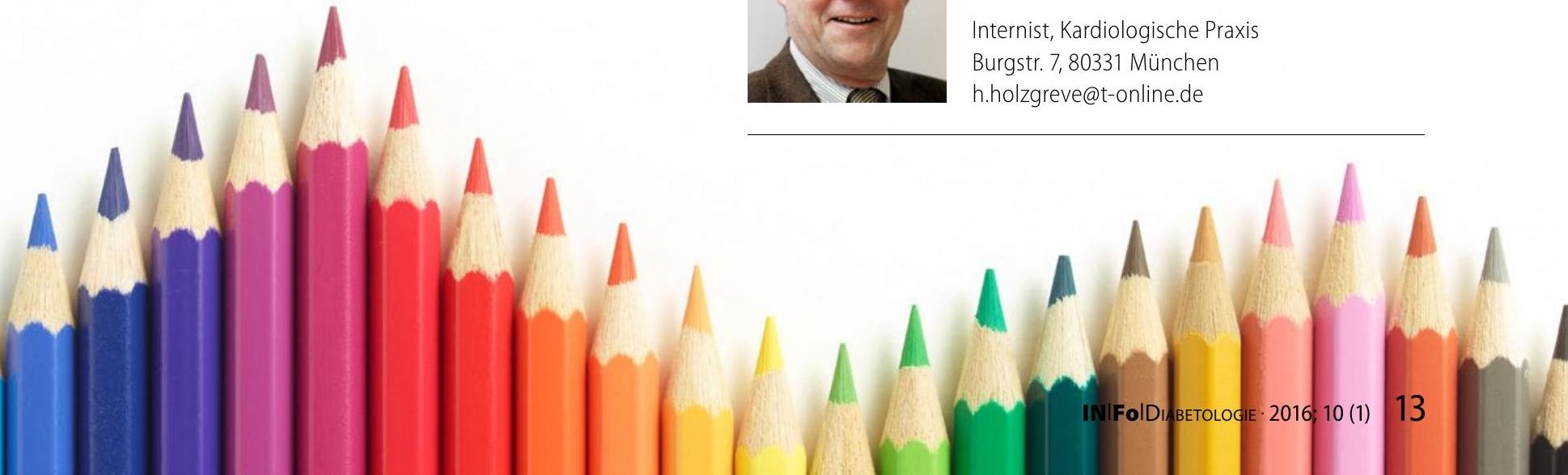

